\title{
Teaching program of breathing coordination in the system of strength training
}

\begin{abstract}
At the time of work with weights during the training, breathing becomes difficult. Therefore, many practitioners hold their breath during the lifting of weights, which leads to an increase in blood pressure in the abdominal cavity and a decrease in blood flow to the brain, resulting in headache, dizziness and weakness. In especially severe cases, a rupture of the blood vessel and the retinal detachment of the eyes may occur, and a rupture of the blood vessel and exfoliation of the retina. In the work is presented the program of teaching the right breathing in the system of exercising the force trainings. Within the program, young women were trained. Three types of respiration were required for development: thoracic (upper), abdominal (middle and lower), mixed (full). The experimental program provided the strengthening of the respiratory muscles by performing exercises according to the method of AN. Strelnikova, as wel as training in coordinating the rhythm of breathing with the rhythm of exercise on the training equipment. In order to determine the effectiveness of the methods developed, it has been carried out a test on physical abilities and physiological possibilities of two women groups, engaged in power-oriented fitness: experimental and control. The introduction of the experimental program into the practice of improving the strength of the experimental program showed positive results in all parameters, however, when comparing the indices of both groups, four out of nine proved to be statistically significant: the p.e.m. of the hands and p.e.m. abdominal press at the level $\rho<0.05$; and two parameters of the Serkin sample: the $2^{\text {nd }}$ and $3^{\text {rd }}$ phases at the level $\rho<0.001$. At the same time, the results of the carried out research allow us to state that the conduct of occupations of the power orientation requires the preliminary formation of the correct/ proper breathing process, which determines the health effect of physical training.
\end{abstract}

Volume 2 Issue I - 2018

\author{
Aftimichuk Olga \\ State University of Physical Education and Sports, Moldova
}

Correspondence: Aftimichuk Olga, State University of Physical Education and Sports, Moldova, Email aftolig@mail.ru

Received: January 19, 2018 | Published: February 20, 2018

Keywords: strength training, breathing, women of young age

\section{Introduction}

The problem of breathing regulation attracted the attention of the largest researchers throughout the entire history of physiology. It was developed mainly in three aspects. The first of them had its main goal to find out why and how the ventilation of the lungs depending on various conditions of human vital activity (motor activity, climbing to the altitude, etc.) changes dramatically in such a way that the optimal partial pressures of oxygen and carbon dioxide in the alveolar air, thus providing the necessary arterial blood gas composition for the body. ${ }^{1}$ The second, also traditional aspect of the problem of breathing regulation is the study of the mechanisms that perform the change in the phases of the respiratory cycle and the "choice" by the body of the optimal values of the frequency and breathing depth. This line of research is known as "breathing self-regulation". ${ }^{2}$ The third aspect of the problem is the localization and functional organization of the respiratory center. ${ }^{3}$

The listed aspects appear to be different sides of a single problem of breathing regulation; however none of them can fruitfully develop without close connection with each others. Respiratory periodicals are vital. The value of ventilation is determined by the gas composition and the concentration of hydrogen ions in the blood and cerebrospinal fluid, as well as the state of the central nervous system. The required volume of ventilation is provided by a complex combination of factors on which the mode of ventilation of the lungs depends. Most importantly, this is important in the system of physical fitness, because proper, trained breathing improves metabolism, causes a healthy appetite and restful sleep, strengthens the immune and nervous systems, thus having a positive effect on the physical and mental health of a person, as well as on mood, determining the factor of "quality of life". The right breathing has a special place in strength training. During exercises with weights, breathing becomes difficult. Uncoordinated breathing with motor acts can lead to loss of orientation in space and concentration of attention on the performance of the exercise.

\section{Materials and methods}

The given position and defined the objectives of our study: the study of the breathing problem in the system of fitness classes; selection of respiratory techniques for the education of right breathing; determination of the level of physical and physiological preparedness of the subjects; development and practical substantiation of the experimental program. The solution of the formulated problems was ensured by a set of appropriate methods, which included: pedagogical observations, testing of physical abilities (strength endurance of the muscles of the hands, legs, abdominals, back) and physiological capabilities (Serkin's test, ${ }^{4}$ Genci's test) ${ }^{5}$ of subjects, pedagogical experiment, methods of mathematical processing and interpretation of statistical data (Table 1).

\section{Serkin's test}

The first phase determine time of breath-holding in inspiration phase in the sitting position; second phase determine time of breathholding in inspiration phase straight after 20 squatting in $30 \mathrm{sec} . ;$ third phase after $1 \mathrm{~min}$. repeat the first phase. 
Table I Assessment of Serkin's test

\begin{tabular}{llll}
\hline Examine contingent & Phases & & \\
& $\begin{array}{l}\mathbf{I}^{\text {st }} \text { phase } \\
\text { Healthy, not trained }\end{array}$ & $\mathbf{2}^{\text {nd }}$ phase & $\mathbf{3}^{\text {rd }}$ phase \\
Healthy, trained & $35-45 \mathrm{sec}$. & $30-50 \%$ of $I^{\text {st }}$ phase & $70-100 \%$ of $I^{\text {st }}$ phase \\
With latent circulatory insufficiency & $45-60 \mathrm{sec}$ & more than $50 \%$ of $I^{\text {st }}$ phase & more than I00\% of $I^{\text {st }}$ phase \\
\hline
\end{tabular}

\section{Genci's test (or respiration stop in expiration phase)}

The tested man/woman in sitting position after 3-5 min. rest do deep expiration and deep inspiration then stop breathing after deep but not maximum expiration with close mouth and hold one's nose. Stopwatch used; normal breath-holding is $25-30 \mathrm{sec}$., well trained sportsmen: 30-90 sec.

\section{Subjects}

The experiment involved women of young age from the fitness club Bella, Chisinau city. The experimental group consisted of 12 women; the control group consisted of 11 people.

\section{Procedures}

The study was conducted in two stages. The first stage included the determination of the homogeneity of the groups under study using methods for testing physical abilities and physiological capabilities of those engaged in the young age. At this stage, the exercise training system introduced a methodology for training respiratory coordination in order to determine the degree of its impact on the dynamics of the physiological and physical development of the experimented. At the second stage of the study, the effectiveness of the elaborated methodology was developed for training exercises of power orientation with the help of repeated testing of examiners.

\section{Statistical analysis}

The processing of the obtained results of the research was carried out by means of mathematical processing and interpretation of statistical data: arithmetical mean the mean $(\bar{x})$, standard deviation $(\delta)$, the standard error of the mean value (s), the reliability of the differences in the mean values of the two empirical sets was determined by the Student's t-criterion.

\section{Description of the experiment}

With strength training, when working with weights, breathing becomes difficult. Consequently, many practitioners hold their breath while lifting weights, which leads to a significant increase in blood pressure in the abdominal cavity and a decrease in blood flow to the brain, resulting in headache, dizziness and weakness. In severely severe cases, a rupture of the blood vessel and exfoliation of the retina can occur. To arrive at the achieving of the best results, we propose a training program for the coordination of breathing developed during the exercises practicing for the development of strength abilities.

The experimental program provides three stages:

A. Stage 1 was oriented to bring up the right breathing. To do this, within the training were introduced exercises to develop 3 types of breathing: thoracic (upper), abdominal (middle and lower), mixed (full). The duration of this stage was two weeks.
B. Stage 2 provided the strengthening of the respiratory muscles by performing exercises according to the method of AN Strelnikova. ${ }^{6,7}$ For the development of respiratory muscles was given three weeks.

C. Stage 3 is the main one, where took place the training of coordination of the breathing rhythm with the rhythm of exercise on the training equipment. It took eight weeks to master this type of coordination; the process of improvement continued for the next 15 weeks of health-training sessions.

\section{Staged content of training}

At the first stage, the preparatory part of the health-strength training lasted 10-12minutes and included general development exercises, performed from various initial positions, taking into account the formation of three types of breathing: thoracic, abdominal and mixed. The main part (35-40min) of health training was directed to work with own weight, using various expander, weighting and dumbbells of small scales. During the work, the rhythm of breathing and its depth were worked out. Muscular work has always been accompanied by an increase in pulmonary ventilation, which is necessary to meet the need for increased oxygen demand and the removal of excess carbon dioxide from the body. The function of the apparatus of external respiration is very closely related to the function of skeletal muscles. During muscular work, pulmonary ventilation can be increased $10-20$ times, reaching $80-1001 / \mathrm{min}$ for an untrained person at $5-81 / \mathrm{min}$ at rest. ${ }^{8}$ This increase is ensured by both increasing and by deepening the respiratory movements. The final part (5-8 minutes) was given to stretching and breathing exercises when performing fixed poses.

The preparatory part of the sessions of the second stage contained general development exercises performed with directed respiratory acts according to the method of A.N. Strelnikova, ${ }^{6,7}$ implying the development of muscles involved in respiration processes. The main part of the training included the content of the first stage, with the only difference being that the weighting agents and dumbbells were of higher weight. Individually every week the weight increased by $0.5-1.0 \mathrm{~kg}$. The final part remained unchanged both in duration and in content. At the third stage, the preparatory part of the exercises was performed according to the traditional method, including a set of general development exercises with directional breathing during their execution. The main part was devoted to work on simulators for the development of various muscles and muscle groups. During the exercises, strict self-control as well as control by the instructor over the compliance of acts of exhalation and inspiration for moments of muscle tension and relaxation was observed. The final part involved stretching for the muscles and muscle groups that participated in each particular workout. Exercises were performed to restore the rhythm of breathing.

To determine the effectiveness of the developed methodology, it 
were tested the physical abilities and physiological capabilities of two groups of women engaged in strength fitness training: experimental and control. The experimental group worked according to our program, which presupposes purposeful training for proper breathing during the performance of forceful exercises, with subsequent control of its execution. The control group was involved in a program that did not require special training for proper breathing.

\section{Results}

An analysis of the results showed that both groups achieved a positive effect from the classes, but at different levels (Figures 1) (Figure 2). In the experimental group of 9 indicators of the tested parameters (Figure 1), 7 demonstrated a high level of statistical reliability $(\rho<0.001)$. The " $1^{\text {st }}$ phase" of the Serkin's test $(t=4.21)$ and the "Genci's test" $(\mathrm{t}=3.98)$ revealed the level of reliability of differences in the initial and final data for $\rho<0.01$. This suggests that targeted training for proper breathing contributes not only to the development of respiratory muscles and the strengthening of the physical functions of the cardio-respiratory system, but also reveals its beneficial effects on the development of physical abilities.
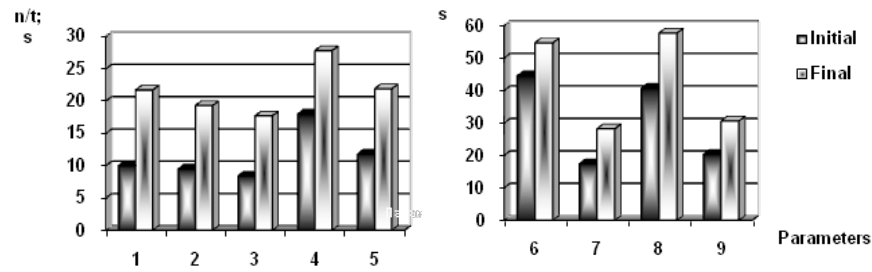

Figure I Dynamics of the average group indicators of the tested parameters of experimental group.

Note I, Power endurance of the Muscles p.e.m. of the hands; 2, p.e.m. of the right foot; 3, p.e.m. of the left foot; 4 , p.e.m. of the abdominal press; 5 , p.e.m. of the back; 6-8, Serkin's test; 9 , Genci's test; n/t, Number of times; s, seconds.

The results of the tested physical abilities and the physiological capabilities of the control group also show statistical certainty. However, their values are located at lower levels (Figure 2). Parameters such as strength endurance of the muscles of the hands $(\mathrm{t}=3.69)$ and legs, right $(\mathrm{t}=4.16)$, legs, left $(\mathrm{t}=4.12)$ demonstrated a level of statistical significance equal to $\rho<0.01$. The other six parameters showed the level of reliability of differences between the initial and final data at $p<0.05$. A comparative analysis of the experimental and control group data was made after seven months of training sessions (the end of the pedagogical experiment was due to the exercise finish of several subjects). Investigated women of both groups increased their physical abilities and physiological capabilities (Table 2).
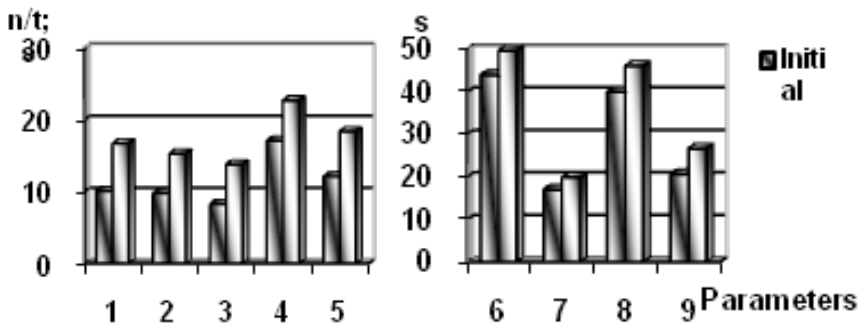

Figure 2 Dynamics of the average group indicators of the tested parameters of the control group.

Note I, Power endurance of the muscles p.e.m. of the hands; 2, p.e.m. of the right foot; 3 , p.e.m. of the left foot; 4 , p.e.m. of the abdominal press; 5 , p.e.m. of the back; 6-8, Serkin's test; 9 , Genci's test; $n / t$, number of times; s, seconds

When comparing their final indices, the statistical reliability $(\rho>0.05)$ did not yield the parameters of the muscle strength of the legs, "right" ( $\mathrm{t}=2.05)$; "feet, left" ( $\mathrm{t}=1.95)$; "back" $(\mathrm{t}=2.0)$; "stage I" of the Serkin's test $(\mathrm{t}=1.92)$ and "Genci's test" $(\mathrm{t}=1.63)$, which, in our opinion, determines the productivity of both strength training programs. However, four indicators revealed the statistical reliability of the final data of the study groups, thus ascertaining the advantage of the experimental technique. At the same time, the strength endurance indices of the muscles of the hands $(\mathrm{t}=2.69)$ and the abdominal press $(t=2.23)$, with $\rho<0.05$, allow us to declare the need for correct breathing during the performance of strength exercises, especially for these exercises muscle groups. The indicators "Stage 2" $(\mathrm{t}=4.45)$ and "Stage 3" $(\mathrm{t}=4.38)$ of Serkin's test demonstrated a high level of statistical reliability of differences in the end-points of both groups $(\rho<$ 0.001 ). This can be explained by the fact that the effect of exercises with specially directed respiratory exercises manifested itself not only in strengthening the respiratory muscles and improving the function of breathing, but also in improving the functions of restoring the body after physical work, which also speaks for the experimental technique.

Table 2 Dynamics of the average group indicators of the tested qualities of the experimental $(n=\mid 2)$ and control groups $(n=I I)$

\begin{tabular}{|c|c|c|c|c|c|c|c|c|c|}
\hline Parameters & & & Groups & $\bar{X} \pm m_{\bar{x}}$ & ti & $\rho \mathbf{i}$ & $\bar{X} \pm m_{\bar{x}}$ & tf & $\rho f$ \\
\hline \multirow[t]{8}{*}{ Physical abilities } & $\begin{array}{l}\text { Strength } \\
\text { Endurance Muscles }\end{array}$ & Hands (q-ty) & $E$ & $10.0 \pm 1.32$ & 0.05 & $>0.05$ & $21.7 \pm 1.47$ & 2.69 & $<0.05$ \\
\hline & & & $\mathrm{C}$ & $10.1 \pm 1.38$ & & & $16.7 \pm 1.14$ & & \\
\hline & & right & $E$ & $9.5 \pm 1.41$ & 0.16 & $>0.05$ & $19.3 \pm 1.62$ & 2.05 & $>0.05$ \\
\hline & & (q-ty) & C & $9.8 \pm 0.76$ & & & $15.3 \pm 1.08$ & & \\
\hline & & left & $E$ & $8.4 \pm 1.5$ & 0.06 & $>0.05$ & $|7.7 \pm 1.4|$ & 1.95 & $>0.05$ \\
\hline & & & C & $8.3 \pm 0.85$ & & & $13.8 \pm 1.03$ & & \\
\hline & & Abdominal Press (q-ty) & $E$ & $18,0 \pm 1,52$ & 0.51 & $>0.05$ & $27.8 \pm 1.52$ & 2.23 & $<0.05$ \\
\hline & & & $\mathrm{C}$ & $17.1 \pm 1.55$ & & & $22.7 \pm 1.31$ & & \\
\hline
\end{tabular}


Table Continued....

\begin{tabular}{|c|c|c|c|c|c|c|c|c|c|}
\hline \multirow[t]{2}{*}{ Parameters } & & & Groups & $\bar{X} \pm m_{\bar{x}}$ & ti & $\rho \mathbf{i}$ & $\bar{X} \pm m_{\bar{x}}$ & tf & $\rho f$ \\
\hline & & & $\mathrm{C}$ & $12.1 \pm 1.55$ & & & $18.4 \pm 1.38$ & & \\
\hline \multirow[t]{6}{*}{$\begin{array}{l}\text { Physiological } \\
\text { Possibilities }\end{array}$} & Serkin's Test (s) & Ist stage & $\mathrm{E}$ & $44.5 \pm 1.69$ & 0.27 & $>0.05$ & $54.7 \pm 1.73$ & 1.92 & $>0.05$ \\
\hline & & & C & $43.9 \pm 1.49$ & & & $49.7 \pm 1.95$ & & \\
\hline & & & C & $17.0 \pm 0.98$ & & & $19.9 \pm 0.87$ & & \\
\hline & & $\begin{array}{l}3 r d \\
\text { stage }\end{array}$ & $E$ & $40.7 \pm 1.66$ & 0.31 & $>0.05$ & $57.7 \pm 1.91$ & 4.38 & $<0.001$ \\
\hline & & & C & $40.0 \pm 1.57$ & & & $46.1 \pm 1.83$ & & \\
\hline & "Genci's Test" (s) & & $E$ & $20.3 \pm 1.87$ & 0.12 & $>0.05$ & $30.6 \pm 1.79$ & 1.63 & $>0.05$ \\
\hline
\end{tabular}

$t_{i}, t_{f}$, initial and final indicators; student t-criterion; $\rho_{i}, \rho_{f}$, initial and final indicators of the level of reliability; E, experimental group; C, control group

\section{Conclusion and recommendation}

The development and introduction of the experimental program in the health-improving activities of the strength orientation demonstrated positive results in all parameters, however, when comparing the indices of both groups, four out of nine proved to be statistically significant: the strength endurance of the muscles of the abdominal muscles and arms at the level $\rho<0.05$; and two parameters of the Serkin sample: the 2nd and 3rd stages at the level $\rho<0.001$. This way, the results of the carried out research allow us to state that the conduct of occupations of the power orientation requires the preliminary formation of the process of proper/correct breathing, which determines the health effect of physical training. The methods of health-improving breathing that can be used can well be used in the training of high-class athletes in various sports. In our opinion, these methods can be included in the content of the professional training of teachers in physical education and coaches in sports. The use of breathing exercises, physical education and sports classes will help enrich the teachers both in terms of improving their own culture and dealing with them, as well as in the aspect of creative approach to their profession.

\section{Acknowledgements}

None.

\section{Conflict of interest}

Author declares that there is no conflict of interest.

\section{References}

1. Silverthorn DU. Human Physiology: An Integrated Approach, 5th ed. Benjamin Cummings, USA; 2009.

2. West JB. Respiratory Physiology: The Essentials, 9th ed. LWW, USA; 2011.

3. Safonov VA, Tarasova NN. Structural and functional organization of the respiratory center. Human Physiology. 2006;32(1):118-131.

4. Pirogova LA, Khovanskaya GN. Basics of medical rehabilitation and non-medicamental therapy: manual. Grodno State Medical University, 2009. p. 21-22.

5. Monakova E. Anatomy and physiology of movements Part 2(2). Lulu Press, USA; 2015. p. 140-141.

6. Alexandra N. Strelnikova Breathing Exercises for Singing (Review) 1950.

7. Paradoxical breathing exercises Strelnikova: Course of lectures on Valeology. Health and traditional Medicine. 2010.

8. Breslav IS, Nozdrachev AD. Regulation of respiration: visceral and behavioral components. Successes of physiological sciences. 2007;38(2):26-45. 